\title{
Improving Hydrostability of ZIF-8 Membranes via Surface Ligand Exchange
}

\author{
Huifeng Zhang ${ }^{\mathrm{a}, \mathrm{b}, \mathrm{c}}$, Joshua James ${ }^{\mathrm{c}}$, Man Zhao ${ }^{\mathrm{b}}$, Ying Yao ${ }^{\mathrm{b}}$, Yushan Zhang ${ }^{\mathrm{b}}$, Baoquan Zhang ${ }^{\mathrm{a}}$, Y.S. Lin ${ }^{\mathrm{c}, *}$ \\ a- School of Chemical Engineering and Technology, Tianjin University. Tianjin, China 300072 \\ b- Institute of Seawater Desalination and Multipurpose Utilization,(SOA) Tianjin. Tianjin, China 300192 \\ c- School for Engineering of Matter, Transport and Energy, Arizona State University, Tempe, Arizona, \\ United States 85287 \\ *- Corresponding Author; email: Jerry.Lin@asu.edu; phone: 001-480-458-8215
}

\begin{abstract}
Zeolitic imidazolate frameworks (ZIFs) are promising materials for gas and liquid separations due to their unique pore structures and tunable surface properties. Recent studies show that ZIF crystalline powders, especially membranes, are not stable in aqueous solutions. The present work shows the improvement of ZIF-8 crystal and membrane stability in water through utilization of a ligand exchange post-modification method which replaces methylimidazole ligands on the outer surface of ZIF-8 crystals or membranes by the more hydrophobic, bulkier 5,6-dimethylbenzimidazole. Ligand exchange modification does not change the crystal structure, morphology or gas permeance as shown ZIF-8 powder and membrane characterization experiments. The modified ZIF-8 powders and membranes retain identical morphologies and crystallinities after static and dynamic water immersion. The modified ZIF-8 membrane exhibits stable water pervaporation flux controlled by the ZIF-8 layer while the unmodified ZIF-8 membrane experiences dissolution of the ZIF-8 layer during water pervaporation. This ligand exchange strategy enables hydrostable ZIF-8 membranes for practical applications involving aqueous solutions.
\end{abstract}

Keywords: Zeolitic imidazolate frameworks, stability, improvement, ligand exchange, ZIF-8 


\section{Introduction}

Zeolitic imidazolate frameworks (ZIFs) are a subclass of microporous metal organic frameworks (MOFs) constituted by divalent transition metal cations with organic imidazole linkers. ZIFs usually take on zeolite topology structures due to the similar chemical bond angle between metal-imidazole-metal in ZIFs and Si-O-Si in zeolites. As opposed to the use of organic template reagents in zeolite synthesis, the imidazolate linkers and solvents employed in synthesis of ZIFs usually offer a structure directing effect on ZIF formation [1, 2]. The self-assembly nature, structural versatility as well as tunable surface properties make ZIFs promising materials for a wide variety of applications including gas separation and storage [3-6], reaction catalysis [7], and sensors [8]. Synthesis and applications of both ZIF powders and membranes have been extensively studied. To date, ZIFs of different crystal sizes and morphologies can be synthesized by either solvothermal methods with organic solvents (e.g. dimethylformamide and methanol) [9, 10] or hydrothermal protocols with water as solvent [11-15].

Membranes of several ZIFs, in particular ZIF-8, were successfully prepared either on inorganic supports [5, 16-19] or on organic supports [20, 21]. Most studies on ZIF-8 membrane were focused on applications for gas separation such as hydrogen separation [18, 21-23] and propylene/propane separation [24-27]. But recent studies have shown growing research efforts devoted to extending applications of ZIF-8 membrane to liquid phase such as organic solvent separation and aqueous mixture separation. Caro and co-workers [28] reported n-hexane/benzene and n-hexane/mesitylene separation by ZIF-8 membranes. ZIF-8 structure are expected to offer excellent ion rejection in water desalination due to the cut-off pore size of ZIF-8 (0.42 nm [29]) between water molecules $(0.276 \mathrm{~nm})$ and most of hydrated salt ions $(0.66-0.76 \mathrm{~nm})$ [30] in saline waters. Jiang and co-workers [31, 32] simulated desalination behavior of ZIF-8 structure in reverse osmosis process, and $100 \%$ rejection of ions in $0.5 \mathrm{M} \mathrm{NaCl}$ solution were achieved. Duke and co-workers [33] explored the potential of tubular alumina tube supported ZIF-8 membranes for saline water desalination via reverse osmosis. They reported an extremely high 
flux of $600 \mathrm{~kg} / \mathrm{m}^{2} . \mathrm{h}$, but a low salt rejection of $6 \%$ possibly due to the membrane defects. In contrast, Huang and co-workers [34] reported water flux of $5.8 \mathrm{~kg} / \mathrm{m}^{2} . \mathrm{h}$ at $25{ }^{\circ} \mathrm{C}$ and high ion rejection of over $99.8 \%$ in seawater desalination using a polycrystalline ZIF-8 membrane on polydopamine modified alumina disks. These results indicate the potential application of ZIF-8 in liquid separations in spite of moderate selectivity being reported in most studies [35].

ZIFs were perceived as intrinsically robust relative to other MOFs due to ZIF's hydrophobic nature and stronger coordination bond between the metal center and anionic nitrogen atoms in imidazolate linkers [1]. Though ZIFs appear stable in gas atmosphere [26] and somewhat chemically harsh conditions [1], increasing evidence shows that ZIFs may not be as stable as they were conceived initially. Yaghi and co-workers [1] reported that ZIF-7 and ZIF-11 remained in the same structure at room temperature or $50{ }^{\circ} \mathrm{C}$ water, but transformed to new crystalline substances in boiling water. Yang and co-workers [36] reported that hydrothermal treatment at lower mass ratio of ZIF powders (ZIF-8, ZIF-7 and ZIF-93) to water (0.06\%wt) led to the collapse of ZIF crystalline structure and formation of ZnO. Microporous ROH type ZIF-71 crystals retained its structure in heat treatment in air up to $250{ }^{\circ} \mathrm{C}$; but partially transformed to dense LCS-type ZIF-72 in water at room temperature [37].

Kasik et al. [38] observed no loss in crystallinity for ZIF-68 membranes for 12 months exposure to laboratory atmospheric moisture conditions; but the loss of crystallinity was found after submersion in water at room temperature for 7 days. Furthermore, an unknown substance derived from the phase shift of ZIF-68 powders and membranes was detected after immersion in boiling water. Zhang et al. [39] observed dissolution of both ZIF-8 crystallites and membranes into water at room temperature; and a hydrolyzed impurity crystalline structure different from ZIF-8 was found. Also, Choi and co-workers [40] reported that at high temperature $\left(400{ }^{\circ} \mathrm{C}\right)$ the ZIF-8 structure is less stable in a steam atmosphere than in inert gas (argon) and air. These results suggest that at low temperatures (below $200{ }^{\circ} \mathrm{C}$ [41] ) ZIFs are stable in gas atmosphere, but unstable in aqueous solutions. These results observed show a stability limitation for ZIFs in 
applications associated with aqueous solutions.

Recently, there is growing interest in chemical or structure modification of the imidazolate linkers to prepare ZIFs with tunable aperture size and improved water stability. Nair and co-workers [42] developed a synthetic approach of ZIFs with hybrid linkers by using a mixed linker synthesis solution; the ZIF-8 linker, 2-methylimidazole (MIM), was fractionally substituted by imidazole-2-carboxaldehyde (ICA, ZIF-90 linker) and benzimidazole (BIM, ZIF-7 linker); resulting in ZIFs with hybrid linkers, ZIF-8-90 with larger pore size and more rigid ZIF-7-8 than ZIF-8. A post synthetic strategy termed solvent-assisted linker exchange (SALE) was applied for ZIF structure modification; MIM in ZIF-8 was replaced by imidazole (IM) in $\mathrm{nBuOH}$ [43], leading to increased solvent-accessible volume, and 2-ethyl-imidazole (EIM) in methanol [44], and 4,5-dichloroimidazolate (DCIM) in 1-propanol [37], respectively, while retaining parent ZIF-8 topology structure. DCIM in ZIF-71 was substituted by 4-bromoimidazolate [44]. In these SALE modifications, the daughter linkers are smaller than the aperture size of the parent ZIFs for the easy access into the cages of framework. In an opposite treatment, Yang and co-workers [36] conducted a linker exchange study on ZIF-8 by using the bulkier 5,6-dimethylimidazolate (DMBIM). They found that the outermost MIM ligands in ZIF-8 crystals were substituted by DMBIM, and ZIF-8 was endowed with better hydrophobic properties. This approach was also applied for partial exchange of MIM ligand in ZIF-8 by ICA, which results in more rigid polymer chains between polyimide matrix and ZIF-8 particles when used as $\mathrm{N}_{2} / \mathrm{NF}_{3}$ separation membrane [45].

Recently Lin and co-workers [39] found that both ZIF-8 crystals and ZIF-8 membrane degrade in water due to the dissolution of ZIF-8 even at room temperature. The degradation rate increases with lower ZIF-8 mass to water ratio or 2-methylimidazolate concentration in the aqueous solution. Thus, ZIF-8 membranes are even less stable than ZIF-8 crystals in aqueous solution. The objective of this work is to study post-synthesis modification on the surface of ZIF-8 membranes (and crystalline powder) by the surface ligand exchange approach using 
hydrophobic DMBIM ligands to improve the water stability of ZIF-8 membranes. Its effect on ZIF-8's hydrophobicity and water stability was examined in comparison with those of pristine ZIF-8 powders and membranes by both static water immersion and dynamic water pervaporation tests.

\section{Experimental}

\subsection{Synthesis and modification of ZIF-8 powders and membranes}

All chemicals were purchased from commercial suppliers and used as received without further purification. $\quad \mathrm{Zn}\left(\mathrm{NO}_{3}\right)_{2} \cdot 6 \mathrm{H}_{2} \mathrm{O} \quad(>98 \%)$, 2-methylimidazole $\quad(\mathrm{MIM},>99 \%)$, 5,6-dimethylbenzimidazole (DMBIM, >99\%) and triethylamine (TEA, >99\%) were purchased from Sigma-Aldrich. Methanol (analytical grade, >99.5\%), $\mathrm{Zn}(\mathrm{OH})_{2}(>99.0 \%)$ were supplied from Sinopharm Chemical Reagents and $\mathrm{NaCOOH}(>99.5 \%)$ from Tianjin Fuchen Chemical Reagents, respectively. The a-alumina powder used to prepare supports for ZIF-8 membrane was A16 from Almaitis, USA.

The parent ZIF-8 powders, later subjected to modification were synthesized in methanol under solvothermal conditions. $1.76 \mathrm{~g} \mathrm{Zn}\left(\mathrm{NO}_{3}\right)_{2} \cdot 6 \mathrm{H}_{2} \mathrm{O}$ was dissolved into $20 \mathrm{~mL}$ methanol, 0.97 g MIM and $0.40 \mathrm{~g} \mathrm{NaCOOH}$ were dissolved in another $20 \mathrm{~mL}$ methanol. The clear MIM solution was poured into zinc solution. The mixed solution was transferred into a glass vial, which was then sealed and heated to $60{ }^{\circ} \mathrm{C}$, and maintained at this temperature for $24 \mathrm{~h}$ for formation of ZIF-8 crystals. At the end of the synthesis process, the precipitated white powders in the synthesis solution were collected by 3 cycles of centrifugation at $9500 \mathrm{rpm}$ for 10 minutes and washing with fresh methanol, followed by drying at $60{ }^{\circ} \mathrm{C}$ under reduced pressure for $24 \mathrm{~h}$. ZIF-8 membranes on a-alumina supports were prepared by the seeded secondary growth method reported previously [39]. The prepared ZIF-8 powders and membranes were stored in a desiccator.

The post-synthesis modification of ZIF-8 powders and membranes was conducted by the 
surface linker exchange method originally reported by Yang and co-workers [36]. For ZIF-8 powder modification, a given amount of ZIF-8 powder was dispersed in half of the required amount of methanol under sonication. DMBIM was fully dissolved in another half of the required amount of methanol, followed by addition of TEA. The ZIF-8 suspension and DMBIM-TEA solution were mixed in a glass vial under stirring, then subject to solvothermal treatment at $60{ }^{\circ} \mathrm{C}$ for $24 \mathrm{~h}$. The weight ratio of ZIF-8 powder: DMBIM: TEA: methanol was 1:1:1:160. The modified powders were washed with methanol and collected by centrifugation followed by drying at room temperature under vacuum of $0.05 \mathrm{MPa}$. For ZIF-8 membrane modification, $0.1 \mathrm{~g}$ DMBIM and $0.1 \mathrm{~g}$ TEA were dissolved into $20 \mathrm{~mL}$ methanol in a glass vial. A dried ZIF-8 membrane was placed into the DMBIM solution with the ZIF-8 layer facing upwards. The vial was then sealed and heated to $60{ }^{\circ} \mathrm{C}$ for $24 \mathrm{~h}$. The modified membrane was taken out and rinsed with methanol and dried at room temperature under vacuum.

\subsection{Characterization}

The crystallinity of ZIF-8 powders and membranes were examined by X-ray diffraction on a X-ray diffractometer (Bruker D8 Advance) in the $2 \theta$ range of $5^{\circ}-50^{\circ}$ at scan speed of $5 \% \mathrm{~min}$ using $\mathrm{Cu} \mathrm{Ka}$ radiation $(\lambda=1.54 \AA$ ) at $40 \mathrm{kV}$ and $40 \mathrm{~mA}$. Scanning electron microscopy (SEM) micrographs of ZIF-8 powders and membranes were taken on a Nova NanoSEM 450 system after gold deposition. Diffuse reflectance Fourier transform infrared spectroscopy (FT-IR) spectroscopy analysis was used to characterize the change in chemical functional groups of ZIF-8 structure before and after modification using a Bio-Rad FTS 6000 Spectrometer within the

wavenumber range of $600 \mathrm{~cm}^{-1}-4000 \mathrm{~cm}^{-1}$. For membrane analysis, the ZIF-8 layer was scraped off from alumina support with a drawknife. Microporosity of the pristine and modified ZIF-8 powders and their respective products after water treatments were characterized by nitrogen physisorption on a Micromeritics ASAP $2020 \mathrm{HD} 88$ after activation at $150{ }^{\circ} \mathrm{C}$ for $12 \mathrm{~h}$ under vacuum. The integrity of as-prepared and modified ZIF-8 membranes was evaluated by measuring singles-gas permeance $\left(\mathrm{He}, \mathrm{CO}_{2}, \mathrm{H}_{2}, \mathrm{O}_{2}, \mathrm{~N}_{2}\right.$ and $\left.\mathrm{SF}_{6}\right)$ on a homemade apparatus. The 
contact angle for ZIF-8 powders and membranes were taken on Kruss PSA 100 Drop Shape Analyzer. The linker exchange degree of MIM in ZIF-8 structure by DMBIM was determined by means of liquid-state proton nuclear magnetic resonance ( $\left.{ }^{1} \mathrm{HNMR}\right)$ of acid digested samples in $\mathrm{DCl} / \mathrm{D}_{2} \mathrm{O}(3: 2$ in volume ratio) on Varian Unity plus-400 spectrometer at $400 \mathrm{MHz}$.

\subsection{Water stability test of ZIF-8 powders and membranes}

Stability tests of the pristine and modified ZIF-8 powders were conducted by suspending the powders in water at a lower ZIF/water weight ratio $(0.06 \mathrm{wt} \%)$ for $24 \mathrm{~h}$. The suspended powders were collected by slowly evaporating up the water at $60{ }^{\circ} \mathrm{C}$, and then subject to characterization. For modified ZIF-8 membranes, the stability tests were conducted in either static immersion in water or dynamic pervaporation test. A modified ZIF-8 membrane was sectioned into pieces and immersed in $40 \mathrm{~mL}$ water, and then taken out for XRD and SEM analysis at specific intervals. The dynamic pure water pervaporation was conducted on modified ZIF-8 membrane at room temperature $\left(22 \pm 2{ }^{\circ} \mathrm{C}\right)$ using same apparatus in our previous work [39]. The morphology and crystalline structure of ZIF-8 membrane before and after pervaporation were measured by XRD and SEM, respectively.

\section{Results and Discussions}

\subsection{Ligand exchange modification on ZIF-8 crystals and membranes}

Post-synthesis modification of surface ligands using the SLER was performed on either ZIF-8 crystallites or membranes by introducing the more hydrophobic DMBIM ligand to the ZIF-8 structure. The prepared parent ZIF-8 crystals show typical ZIF-8 morphologies: well-defined rhombic dodecahedron with truncated corners with average size of ca. $4 \mu \mathrm{m}$, as shown in Fig.1(a). XRD patterns shown in Fig. 2 includes characteristic peaks of the ZIF-8 crystal structure at $2 \theta$ of around $7.3^{\circ}(011), 10.2^{\circ}(002)$ and $12.7^{\circ}(112)$. Fig.3 shows type-I nitrogen adsorption isotherms for parent ZIF-8 powders, which is typical for microporous materials (pore size less than $2 \mathrm{~nm}$ ). The sharp increase in absorbed nitrogen quantity at very low 
relative pressure is due to the existence of intrinsic micropores of ZIF-8 structure. Meanwhile the relative small second uptake at near saturated relative pressure indicates the presence of macroporosity due to the packing of ZIF-8 particles. The BET surface and pore volume of ZIF-8 powders were $1206 \mathrm{~m}^{2} / \mathrm{g}$ and $0.640 \mathrm{~cm}^{3} / \mathrm{g}$ (given in Table 1), respectively, in the typical range of $1000-1600 \mathrm{~m}^{2} / \mathrm{g}$ and $0.58-0.65 \mathrm{~cm}^{3} / \mathrm{g}$ for ZIF-8 powders [12]. The characteristic morphologies, correspondence in crystalline structure as well as microporosity confirm the formation of pure ZIF-8 powders with minimum impurities.
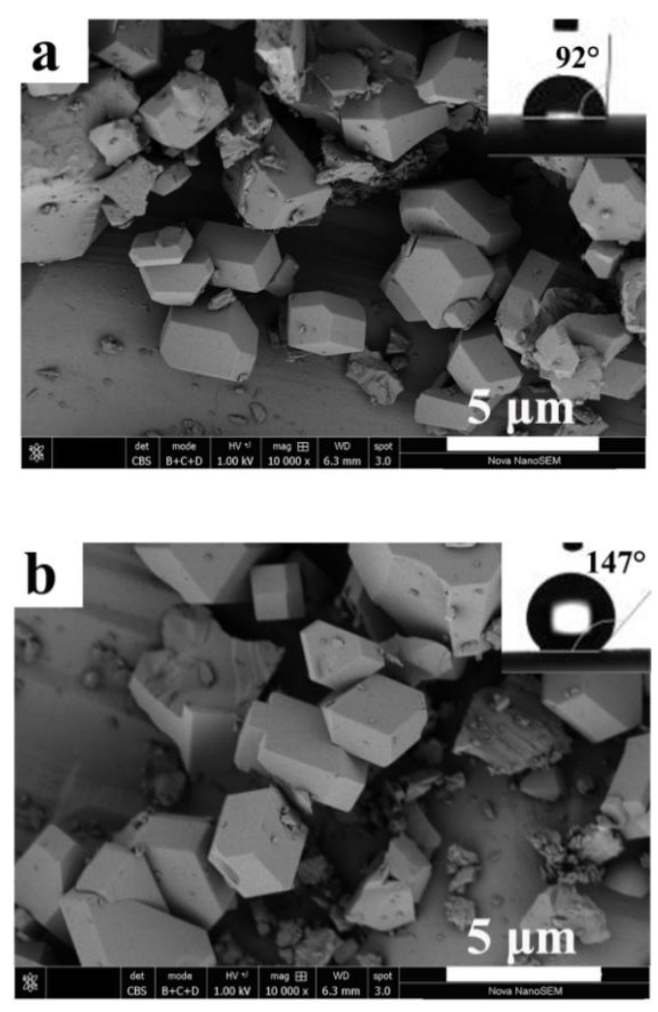

Fig. 1 SEM micrographs of (a) pristine ZIF-8 and (b) modified ZIF-8 (the insets are the corresponding images of static water contact angles)

After subjection to ligand exchange with DMBIM, the obtained ZIF-8 powders retain nearly identical morphology both in shape and size (Fig.1(b)) and undisturbed crystallinity as evidenced by excellent agreement in XRD spectra with parent ZIF-8 (shown in Fig.2). The negligibly reduction in BET surface $\left(1154 \mathrm{~m}^{2} / \mathrm{g}\right)$ and pore volume $\left(0.627 \mathrm{~cm}^{3} / \mathrm{g}\right)$ (in Table 1) 
suggest preservation of the microporous ZIF-8 structure. In addition, after ligand exchange treatment, the white pristine ZIF-8 powders turned to slight beige, the intrinsic color of DMBIM, even after thorough washing with fresh methanol.

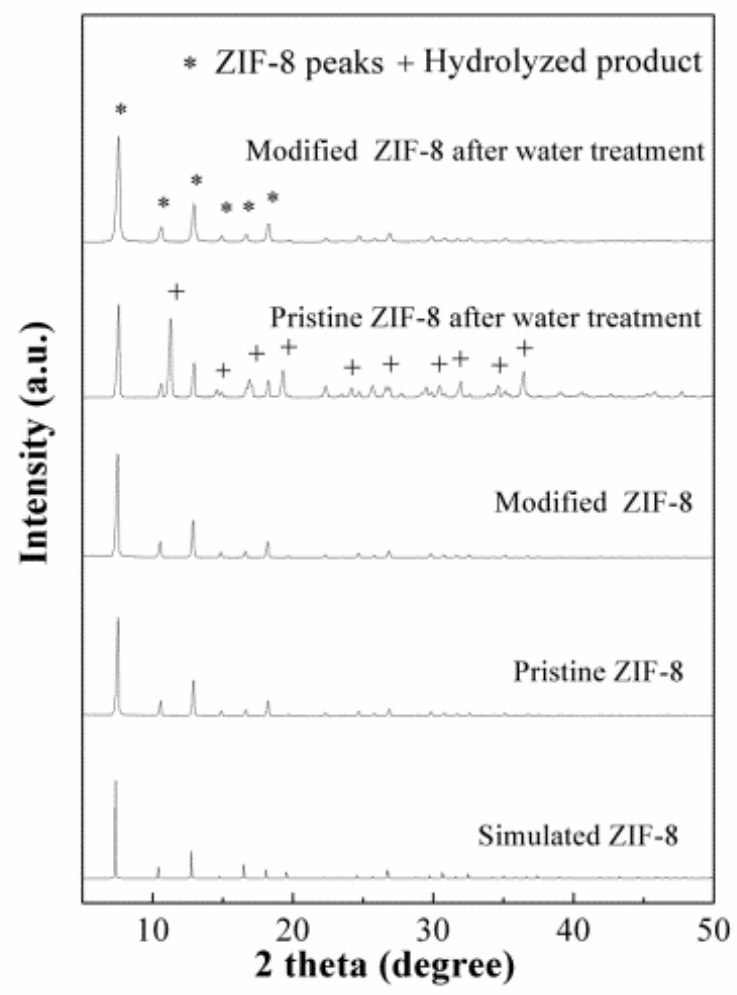

Fig.2 XRD patterns of simulated, pristine, modified ZIF-8 and their corresponding products after water treatment

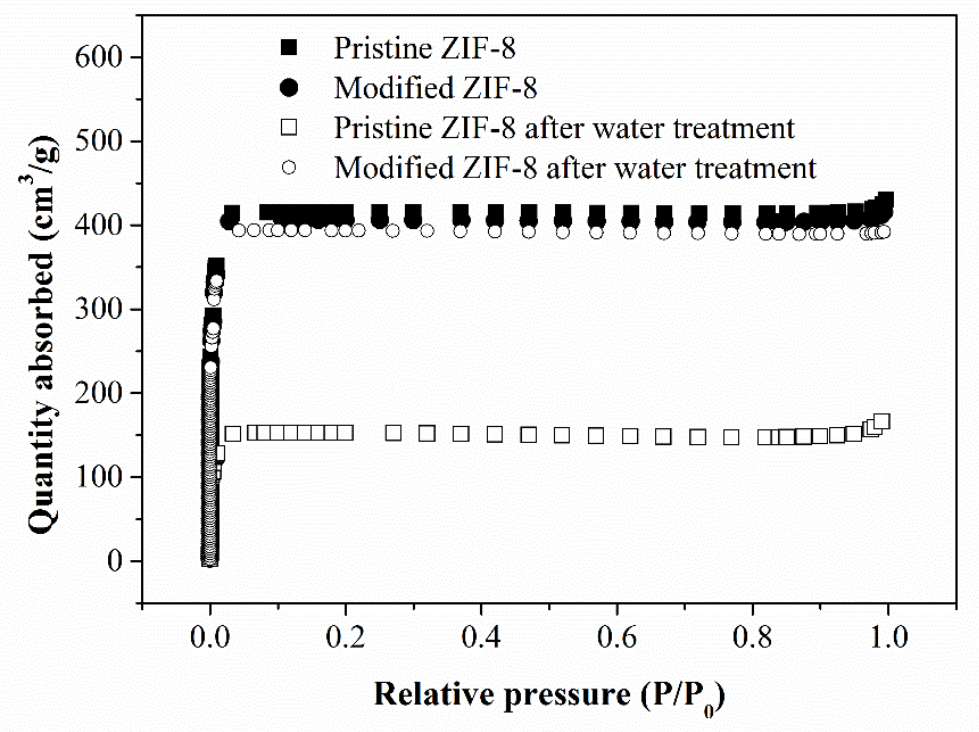

Fig.3 Nitrogen sorption isotherms of pristine, modified ZIF-8 and their corresponding products after 
water treatment

Table 1. BET surface areas and pore volumes of pristine and modified ZIF-8 powders and their corresponding products after water treatment

\begin{tabular}{|c|c|c|c|c|c|c|c|}
\hline \multicolumn{2}{|c|}{ Pristine } & \multicolumn{2}{|c|}{ Modified } & \multicolumn{2}{|c|}{ Pristine in water } & \multicolumn{2}{|c|}{$\begin{array}{l}\text { Modified in } \\
\text { water }\end{array}$} \\
\hline $\begin{array}{c}\mathrm{S}_{\mathrm{BET}} \\
\left(\mathrm{m}^{2} / \mathrm{g}\right)\end{array}$ & $\begin{array}{c}V_{\text {pore }} \\
\left(\mathrm{cm}^{3} / \mathrm{g}\right)\end{array}$ & $\begin{array}{c}\mathrm{S}_{\mathrm{BET}} \\
\left(\mathrm{m}^{2} / \mathrm{g}\right)\end{array}$ & $\begin{array}{c}V_{\text {pore }} \\
\left(\mathrm{cm}^{3} / \mathrm{g}\right)\end{array}$ & $\begin{array}{c}\mathrm{S}_{\mathrm{BET}} \\
\left(\mathrm{m}^{2} / \mathrm{g}\right)\end{array}$ & $\begin{array}{c}\mathrm{V}_{\text {pore }} \\
\left(\mathrm{cm}^{3} / \mathrm{g}\right)\end{array}$ & $\begin{array}{c}\mathrm{S}_{\mathrm{BET}} \\
\left(\mathrm{m}^{2} / \mathrm{g}\right)\end{array}$ & $\begin{array}{c}\mathrm{V}_{\text {pore }} \\
\left(\mathrm{cm}^{3} / \mathrm{g}\right)\end{array}$ \\
\hline 1206 & 0.640 & 1154 & 0.627 & 438 & 0.267 & 1140 & 0.607 \\
\hline
\end{tabular}

$\mathrm{S}_{\mathrm{BET}}$ : Specific surface areas by the BET method

$\mathrm{V}_{\text {pore }}$ : Pore volumes by t-plot method
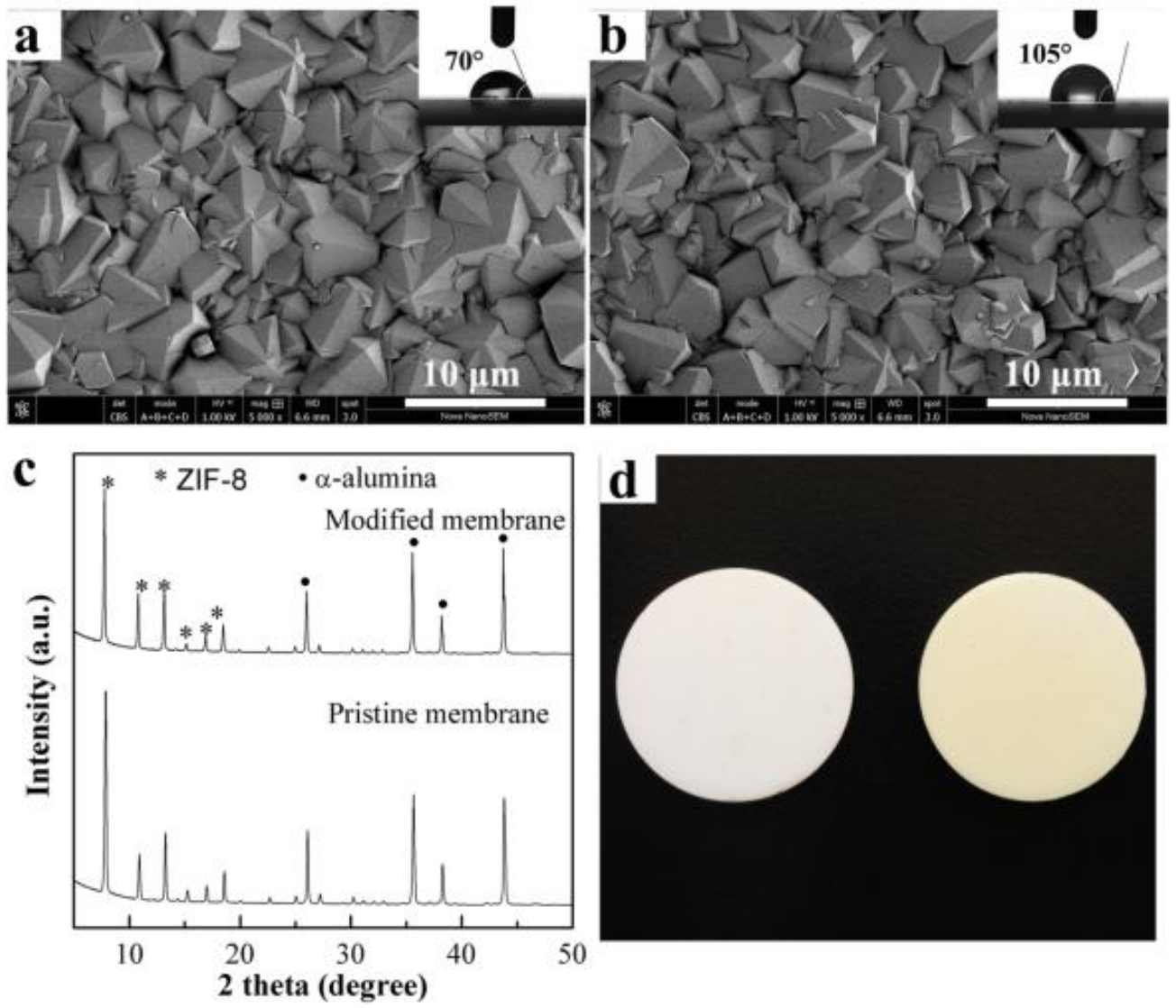

Fig.4 Comparison of ZIF-8 membranes in surface micrographs (a) pristine and (b) modified ZIF-8 membrane, and corresponding static water contact angle (the inset of $(a)$ and $(b)$ ), (c) XRD patterns and (d) appearance change of a ZIF-8 membrane before and after modification

Fig.4 presents the morphologies, XRD patterns and appearances of an a-alumina supported ZIF-8 membrane before and after modification. It is clear that the alumina support is completely covered by homogeneous, well-intergrown ZIF-8 grains with size of ca. $5 \mu \mathrm{m}$, and no cracks and pinholes are visibly observed (Fig.4(a)). The XRD spectrum for pristine membrane shown in 
Fig.4(c) is clearly a combination of ZIF-8 peaks and a-alumina peaks. The XRD pattern, along with morphology, confirms the formation and growth of a pure ZIF-8 structure with a high degree of crystallinity. The unchanged morphology and XRD spectrum after ligand exchange modification were observed as seen from Fig.4(b) and Fig.4(c), indicating that ligand exchange reaction did not disturb the ZIF-8 membranes' crystalline structure. In addition, the enhanced hydrophobicity of the modified ZIF-8 membrane is evidenced by the increase in static water contact angle from $70^{\circ}$ (inset of Fig.4(a)) to $105^{\circ}$ (inset of Fig.4(b)). Furthermore, the modified membrane became slightly beige (Fig.4(d)). These results are similar to those of ZIF-8 powders.

Fig.5 shows IR spectra for pristine ZIF-8 powder and modified ZIF-8 powder and membrane. The IR spectrum of modified ZIF-8 membrane is in good agreement with that of modified ZIF-8 powder. This suggests that the powders scraped off from the alumina supports (ZIF-8 membrane) have the same structure as the modified ZIF-8 crystals. Basically, the IR spectra of modified powders and membrane are dominated by that of reference ZIF-8, implying the ZIF-8 retains same structure after ligand exchange reaction. However, some remarkable peaks are observed in the IR spectra of both modified ZIF-8 powder and the membrane. The 810 $\mathrm{cm}^{-1}$ and $850 \mathrm{~cm}^{-1}$ band in these IR spectra denote the out-of-plane deformation vibrations of $\mathrm{C}-\mathrm{H}$ bond in the phenyl rings of DMBIM (Local enlargement 1 in Fig.5). Meanwhile, two red shifts to lower wavenumbers (Local enlargement 1 in Fig.5) and the disappearance of absorption at $3100 \mathrm{~cm}^{-1}$ (N-H stretching vibration) (Local enlargement 2 in Fig.5) suggest the deprotonation and coordination of nitrogen atoms in DMBIM to zinc ions. These are consistent with the data of Yang and co-workers [36]. However, for the ZIF-8 membrane, the newly emerged absorption bands are rather weak. 


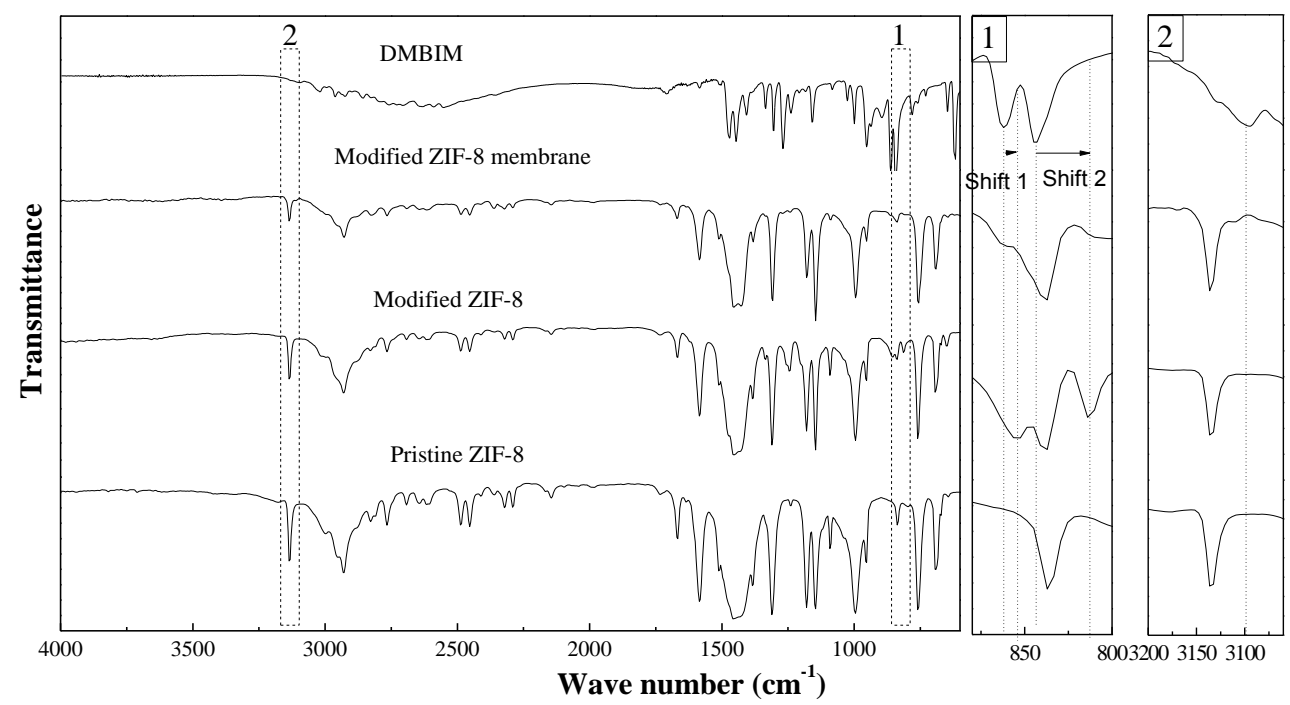

Fig. 5 FT-IR spectra of modified ZIF-8 powders and membrane after modification by ligand exchange (the spectra of pristine ZIF-8 and DMBIM shown as references)

To further confirm the successful exchange of DMBIM with MIM in ZIF-8, ligand exchange molar ratio (DMBIM / (DMBIM + MIM)) was measured by means of ${ }^{1} \mathrm{HNMR}$ analysis. Fig.6 presents the ${ }^{1}$ HNMR spectra of the MIM, DMBIM and digested modified ZIF-8 powder and membrane. The ligand exchange molar ratio for ZIF-8 powders is $4.5 \%$. This value is lower than the exchange degree of MIM ligands in ZIF-8 by DMBIM (9.1\% ) [36] and ICA (24.1-27.9 \%) [45]. Yang and co-workers [36] reported the ligand exchange of bulky DMBIM with MIM in the ZIF-8 structure occurs at the outmost surface of the crystallites. Thus, it can be anticipated that the larger the crystallites are, the lower ligand exchange ratio is in ligand exchange reaction. The ZIF-8 crystal sizes in Ref [36] and Ref [45] are $60 \mathrm{~nm}$ and $100 \mathrm{~nm}$, respectively. So, the lower ligand exchange ratio in this work can be attributed to larger size of parent ZIF-8 crystals ( $4 \mu \mathrm{m}$ in Fig.1(a)). Moreover, though the sizes of crystal grains in ZIF-8 membrane are comparable to ZIF-8 powders, the ZIF-8 membrane gives a lowest ligand change ratio of $1.8 \%$, which is consistent with the weaker characteristic absorption peaks in FT-IR spectrum of ZIF-8 membrane in comparison with that of ZIF-8 crystals. This lower ligand exchange can be reasonably ascribed to the compact intergrowth of ZIF-8 crystallites on the 
alumina support during ZIF-8 membrane formation, which leads to the reduction in external surfaces in contact with reactants during ligand exchange reaction compared with the highly dispersed ZIF-8 particles in solvent. Nevertheless, the combination of FT-IR and ${ }^{1}$ HNMR analyses and increased membrane contact angle indicates the successful introduction of DMBIM onto the ZIF-8 crystals and membrane.

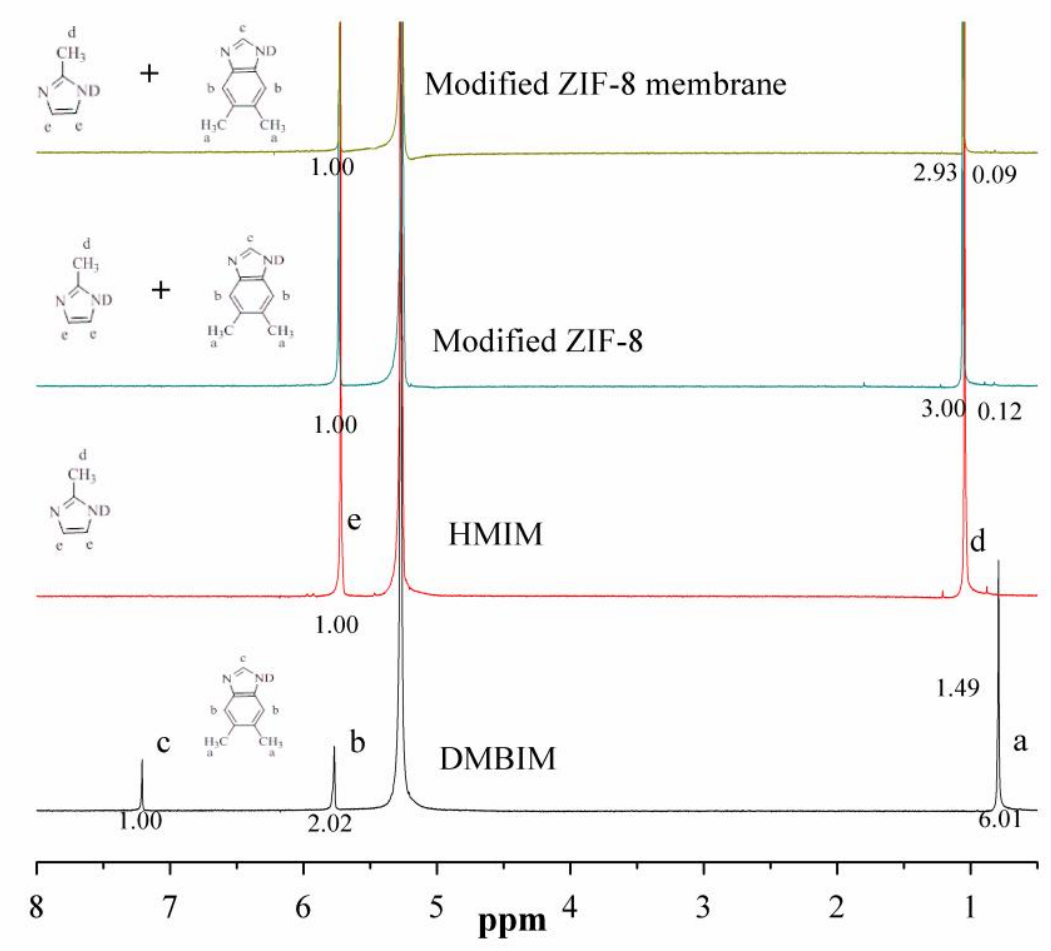

Fig. $6{ }^{1} \mathrm{H}-N M R$ spectra of modified ZIF-8 powders and ZIF-8 membranes after digestion in 3:2 $\mathrm{DCl} / \mathrm{D}_{2} \mathrm{O}$ (volume ratio) mixture. The degree of ligand exchange was obtained from the integrated intensity ratio $(I a+I b) /(I a+I b+I d)$.

For ZIF-8 membrane, the integrity of membrane is of particular importance as surface modification would impair the tight packing of intergrown grains in the ZIF-8 layer. As compared to flexible polymer membrane, the crystalline membrane layer on inorganic support is more fragile and brittle. The ligand exchange modification on membrane might cause stress between highly packed ZIF-8 grains, which may lead to the formation of defects in ZIF-8 layer. This would not be the concern for ligand exchange modification on ZIF-8 powder. The effect of ligand exchange on integrity of membrane was examined by measuring gas permeaces of small 
gases using a ZIF-8 membrane before and after modification. Single gas permeance data for $\mathrm{H}_{2}$, $\mathrm{He}, \mathrm{N}_{2}, \mathrm{O}_{2}, \mathrm{CO}_{2}$ and $\mathrm{SF}_{6}$ shown in Fig.7 for the pristine membrane are comparable with literature data and follow the same trend [26]. Slight increases in the permeance for all single gases were observed after modification treatment. Meanwhile the ideal selectivity of helium over $\mathrm{SF}_{6}$ increased from 138 before modification to 184 after modification, indicating the integrity of the ZIF-8 membrane is even improved after ligand exchange treatment. The ligand exchange modification is more like a rearrangement reaction between the parent and daughter ligands. In the solvent of $\mathrm{MeOH}$, the dissolved DMBIM ligands take MIM's place in ZIF-8. However, during this process, partial decomposition of ZIF-8 is expected, which could result in the reduction in thickness of ZIF-8 layer. This might be the reason for the increase in gas permeances. Furthermore, under solvothermal conditions $\left(60{ }^{\circ} \mathrm{C}\right)$ for ligand exchange reaction, Ostwald-ripening effect may cause growth of the crystal grains in ZIF-8 layer, and hence seal some minor defects of the ZIF-8 layer and improve the membrane's integrity. On the other hand, the replacement of bulkier DMBIM ligands onto ZIF-8 structure is expected to decrease the effective pore sizes, favoring diffusion of small molecules over big ones. These two reasons may be responsible for the increase in helium/SF 6 ideal selectivity of $\mathrm{ZIF}-8$ membrane after ligand exchange treatment.

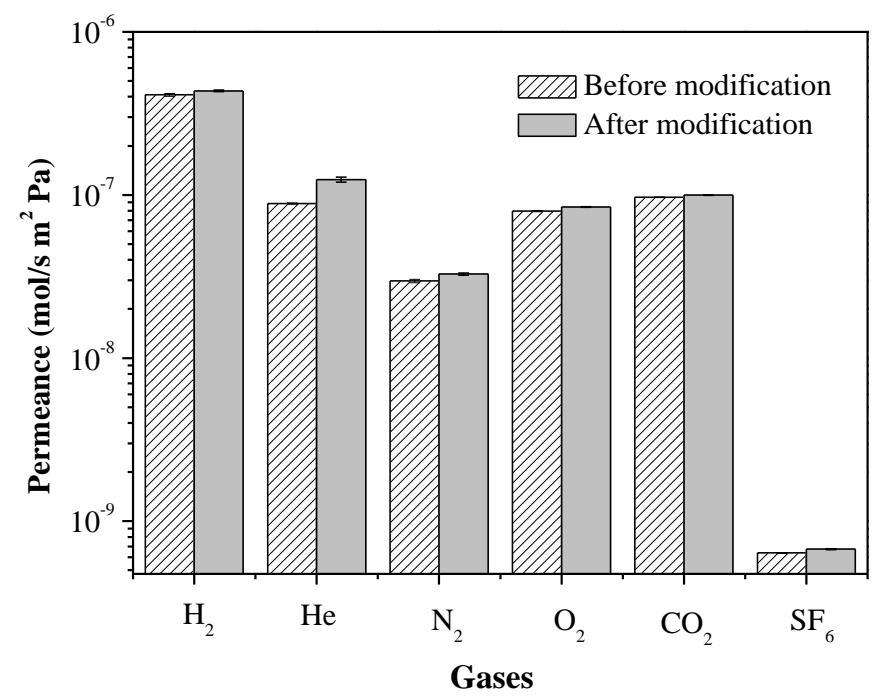

Fig.7 ZIF-8 membrane single gas permeances before and after ligand exchange modification 

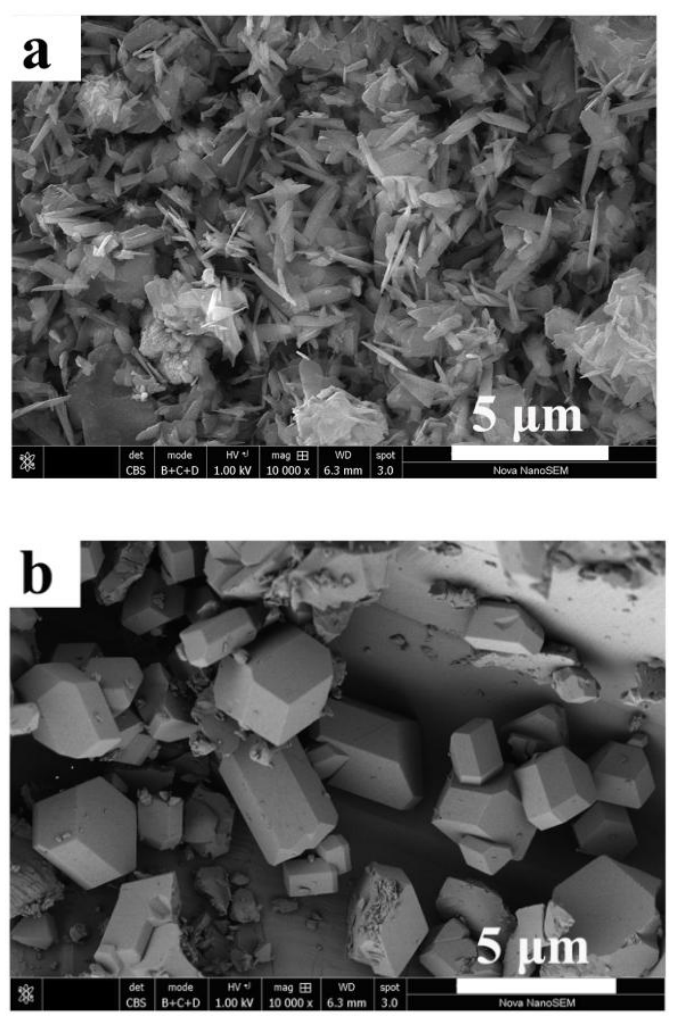

Fig.8 SEM micrographs of (a) pristine ZIF-8 and (b) modified ZIF-8 after water treatment

\subsection{Stability improvement of ZIF-8 powders and membranes}

The ZIF-8 powders before and after immersion in pure water show significantly different morphology and XRD patterns, as shown in Fig.8(a) and Fig.2. Needle-like impurities appear with completely separate set of XRD peaks marked with "+” in XRD pattern in Fig.2 for the ZIF-8 sample after immersion in water. These indicate formation of new different crystalline substance(s). Table 1 shows the significant reduction in BET surface $\left(438 \mathrm{~m}^{2} / \mathrm{g}\right)$ and pore volume $\left(0.264 \mathrm{~cm}^{3} / \mathrm{g}\right)$ for ZIF-8 powders after water treatment. This suggests loss of microporosity of ZIF-8 crystals and a denser structure of the new substance after water treatment. Since no other materials were used in the experiment, this new crystalline product may be formed as a result of interaction between ligands dissolved from ZIF-8 with the water molecules. More study is ongoing to resolve the structure the hydrolyzed crystalline product.

To assess the effect of modification on the water stability, the modified ZIF-8 powders were 
brought to contact with water under similar conditions. The unknown hydrolyzed crystalline structure in observed after immersion of pristine ZIF-8 in water is not observed for the modified ZIF-8 sample. Furthermore, the modified ZIF-8 sample after water treatment has an identical morphology (Fig.8(b), and crystal structure (Fig.2) as the pristine ZIF-8 powder. The microporous properties (Table 1) are essentially unchanged. The slight reduction in surface area might be ascribed to the presence of bulkier DMBIM. In addition, the modified ZIF-8 crystals can float on the surface of water during full test duration while pristine ZIF-8 is dispersed in water after 15 min contact with water. The water contact angle for the modified and unmodified ZIF-8 powders is respectively about $147^{\circ}$ (inset of Fig.1(b)) and $92^{\circ}$ (inset of Fig.1(a)) respectively. These results clearly indicate the enhanced hydrophobicity of modified ZIF-8 due to introduction of the water-repellant DMBIM onto ZIF-8 surface. The improved surface hydrophobicity is responsible for the greater water stability of the modified ZIF-8 crystals.

The static water stability of modified ZIF-8 membrane was evaluated by characterizing the membranes after immersion in water for various durations. SEM images of ZIF-8 membrane surface before and after immersion in water are given in Fig.9 and XRD patterns for the samples after various immersion durations are given in Fig.10. In contrast to the unmodified ZIF-8 membrane which was gradually degraded from alumina support while in immersion in water at room temperature due to the hydrolysis of ZIF-8 structure, the surface of DMBIM modified ZIF-8 membrane remained essentially intact, as evidenced by almost same morphology of surface SEM images before (Fig.9(a)) and after immersion (Fig.9(b)) in water for $132 \mathrm{~h}$. Meanwhile, the corresponding XRD patterns of the modified ZIF-8 membrane show nearly consistent crystal structure both in peak positions and relative intensities (Fig.10). 

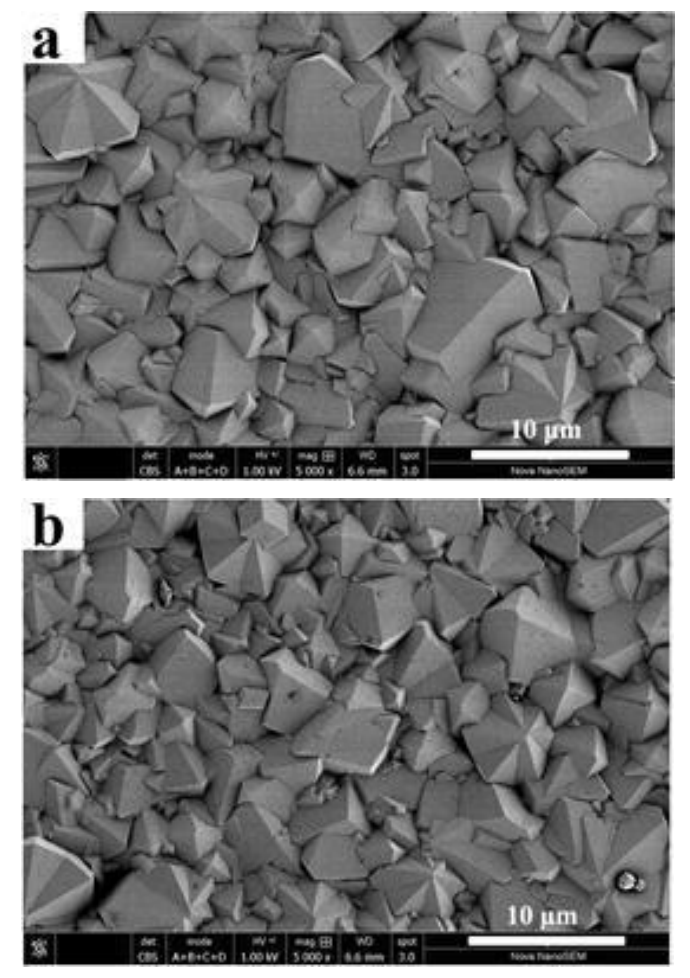

Fig.9 SEM images of a modified ZIF-8 membrane surface before (a) and after (b) immersion in pure water for $132 \mathrm{~h}$

Fig.11 gives the time dependence of water pervaporation flux for the modified ZIF-8 membrane and that for pristine ZIF-8 membrane. For the modified ZIF-8 membrane, the flux starts at ca. $0.15 \mathrm{~kg} / \mathrm{m}^{2} . \mathrm{h}$ during first $4 \mathrm{~h}$, then increases to $0.50 \mathrm{~kg} / \mathrm{m}^{2} . \mathrm{h}$ in the following $6 \mathrm{~h}$. Afterwards, this value kept roughly constant in the rest of pervaporation test. Under the constant driving force (chemical potential difference), water pervaporation flux is related to water solubility and diffusivity in ZIF-8 membrane. The transient increase in water pervaporation flux indicates a gradual change in either water solubility or diffusivity in ZIF-8 eventually reaching the steady state value. A similar trend is observed for the pristine ZIF-8 membrane, which has a lower starting flux of ca. $0.04 \mathrm{~kg} / \mathrm{m}^{2} . \mathrm{h}$ and a much higher stead-state permeation flux of ca. 1.50 $\mathrm{kg} / \mathrm{m}^{2} . \mathrm{h}$ [39] than the modified ZIF-8 membrane. The difference in the initial fluxes for the two ZIF-8 membranes is due to difference in either water solubility or diffusivity in pristine and modified ZIF-8. Our previous work revealed that for the pristine supported ZIF-8 membrane, the alumina supported ZIF-8 layer was almost completely etched off during pervaporation test. 
Therefore, the approximately steady water flux of $1.50 \mathrm{~kg} / \mathrm{m}^{2} . \mathrm{h}$ for pristine ZIF- 8 membrane after $9 \mathrm{~h}$ pervaporation mainly represents the resistance of alumina support to water pervaporation. For the modified ZIF-8 membrane, the final lower water flux is determined by the ZIF-8 layer.

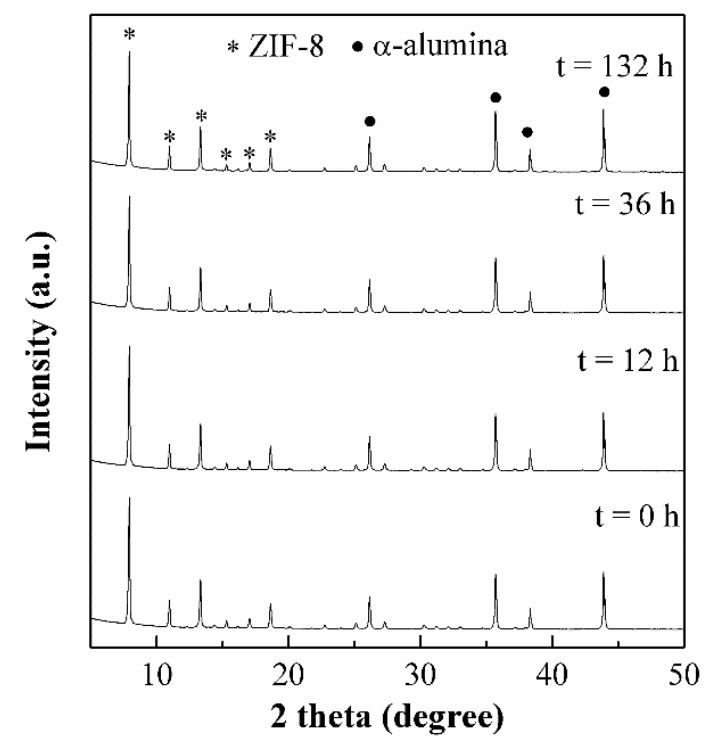

Fig.10 XRD patterns of a modified ZIF-8 membrane after immersion in pure water for various immersion times

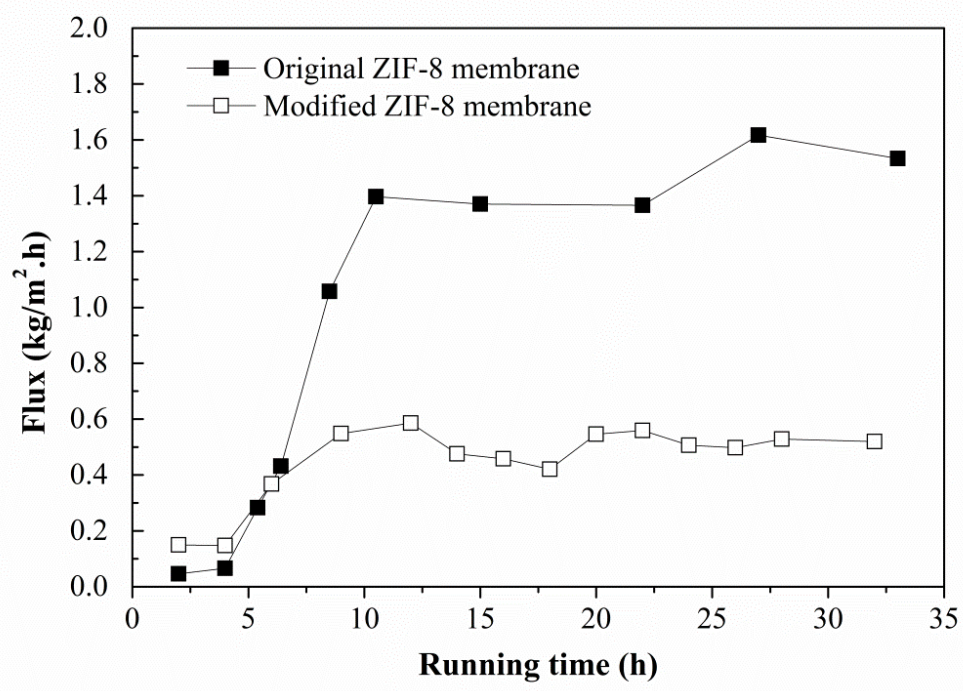

Fig.11 Comparison of time dependence of water pervaporation flux at room temperature between pristine [39] and modified ZIF-8 membranes 

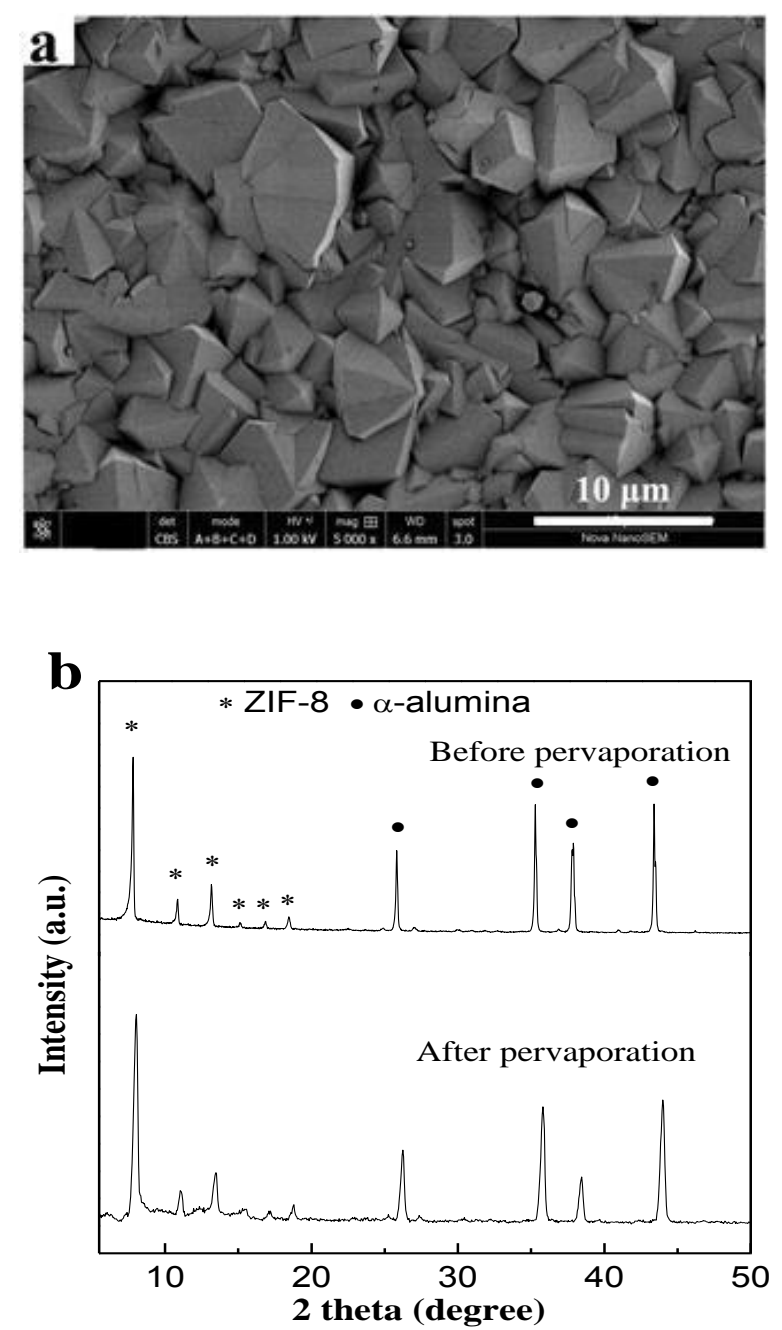

Fig.12 SEM micrograph (a) and XRD pattern (b) of a modified ZIF-8 membrane after pervaporation with pure water at room temperature

Since the modified ZIF-8membrane is derived from the pristine membrane via ligand exchange, the lower constant water flux $\left(0.50 \mathrm{~kg} / \mathrm{m}^{2} . \mathrm{h}\right)$ for the modified ZIF-8 membrane can only be attributed to the additional resistance from the ZIF-8 layer grown on alumina support. In other words, the water flux of $0.50 \mathrm{~kg} / \mathrm{m}^{2} . \mathrm{h}$ reflects the overall water pervaporation resistance from both alumina supports and ZIF-8 layer. Based on the above different steady-state water fluxes, the contribution of ZIF-8 layer to overall resistance is roughly estimated to be about 66.7\%. SEM image and XRD pattern of modified ZIF-8 membrane after pervaporation are shown in in Fig.12, which present an unchanged surface morphology and XRD patterns as compared to membrane before the test. Since the ligand exchange molar ratio for ZIF-8 
membrane is only ca.1.8\% (revealed by ${ }^{1} \mathrm{HNMR}$ in Fig.7), the stability test results indicate that the introduction of even a small amount of DMBIM onto ZIF-8 structure can protect ZIF-8 membrane (both under static and dynamic conditions) against hydrolysis in aqueous solutions. Considering increasing interest in aqueous liquid separation using ZIF membranes, such as alcohol-water separation and water desalination, the ligand exchange modification would provide a viable strategy to address the water stability issue of ZIF memrbanes.

\section{Conclusions}

MIM ligand on the outer surface of ZIF-8 crystals in the powder or membrane can be replaced by the bulkier ligand, DMBIM. This ligand exchange modification does not change the morphology, crystal structure of either ZIF-8 powders or ZIF-8 membranes, but results in a slightly reduced microporosity of ZIF-8 powders and enhanced-integrity of ZIF-8 membrane. The modification results in improvement in the water stability of ZIF-8 powders and membranes due to enhanced surface hydrophobicity. The ligand exchange strategy can be explored to develop stable ZIF powders or membranes for practical applications involving aqueous liquids.

\section{Acknowledgement}

The authors would like to acknowledge the financial support from National Natural Science Foundation of China (No. 21276055), National Science Foundation of the United States (CBET-1511005), National Natural Science Youth Foundation of China (No. 21606059) and Marine Public Welfare Industry Research Project (201505006-3). Huifeng Zhang acknowledges useful discussion with Dr. Xueliang Dong in preparation of this manuscript.

\section{References:}

[1] K.S. Park, Z. Ni, A.P. Cote, J.Y. Choi, R. Huang, F.J. Uribe-Romo, H.K. Chae, M. O'Keeffe, O.M. Yaghi, Exceptional chemical and thermal stability of zeolitic imidazolate frameworks, Proc Natl Acad Sci U S A, 103 (2006) 10186-10191.

[2] X.-C. Huang, Y.-Y. Lin, J.-P. Zhang, X.-M. Chen, Ligand-Directed Strategy for Zeolite-Type Metal-Organic Frameworks: Zinc(II) Imidazolates with Unusual Zeolitic Topologies, Angewandte Chemie, 118 (2006) 1587-1589.

[3] R. Banerjee, A. Phan, B. Wang, C. Knobler, H. Furukawa, M. O'Keeffe, O.M. Yaghi, High-Throughput Synthesis 
of Zeolitic Imidazolate Frameworks and Application to $\mathrm{CO}_{2}$ Capture, Science, 319 (2008) 939-943.

[4] A. Phan, C.J. Doonan, F.J. Uribe-Romo, C.B. Knobler, M. O’Keeffe, O.M. Yaghi, Synthesis, Structure, and Carbon Dioxide Capture Properties of Zeolitic Imidazolate Frameworks, Accounts of Chemical Research, 43 (2009) 58-67.

[5] H. Bux, F. Liang, Y. Li, J. Cravillon, M. Wiebcke, J. Caro, Zeolitic Imidazolate Framework Membrane with Molecular Sieving Properties by Microwave-Assisted Solvothermal Synthesis, Journal of the American Chemical Society, 131 (2009) 16000-16001.

[6] C. Zhang, W.J. Koros, Zeolitic Imidazolate Framework-Enabled Membranes: Challenges and Opportunities, The Journal of Physical Chemistry Letters, 6 (2015) 3841-3849.

[7] C. Chizallet, S. Lazare, D. Bazer-Bachi, F. Bonnier, V. Lecocq, E. Soyer, A.-A. Quoineaud, N. Bats, Catalysis of Transesterification by a Nonfunctionalized Metal-Organic Framework: Acido-Basicity at the External Surface of ZIF-8 Probed by FTIR and ab Initio Calculations, Journal of the American Chemical Society, 132 (2010) 12365-12377.

[8] G. Lu, J.T. Hupp, Metal-Organic Frameworks as Sensors: A ZIF-8 Based Fabry-Pérot Device as a Selective Sensor for Chemical Vapors and Gases, Journal of the American Chemical Society, 132 (2010) 7832-7833.

[9] Y.-R. Lee, M.-S. Jang, H.-Y. Cho, H.-J. Kwon, S. Kim, W.-S. Ahn, ZIF-8: A comparison of synthesis methods, Chemical Engineering Journal, 271 (2015) 276-280.

[10] S.R. Venna, J.B. Jasinski, M.A. Carreon, Structural Evolution of Zeolitic Imidazolate Framework-8, Journal of the American Chemical Society, 132 (2010) 18030-18033.

[11] Y.C. Pan, Y.Y. Liu, G.F. Zeng, L. Zhao, Z.P. Lai, Rapid synthesis of zeolitic imidazolate framework-8 (ZIF-8) nanocrystals in an aqueous system, Chemical Communications, 47 (2011) 2071-2073.

[12] K. Kida, M. Okita, K. Fujita, S. Tanaka, Y. Miyake, Formation of high crystalline ZIF-8 in an aqueous solution, CrystEngComm, 15 (2013) 1794-1801.

[13] S. Tanaka, K. Kida, M. Okita, Y. Ito, Y. Miyake, Size-controlled Synthesis of Zeolitic Imidazolate Framework-8 (ZIF-8) Crystals in an Aqueous System at Room Temperature, Chemistry Letters, 41 (2012) 1337-1339.

[14] A.F. Gross, E. Sherman, J.J. Vajo, Aqueous room temperature synthesis of cobalt and zinc sodalite zeolitic imidizolate frameworks, Dalton Transactions, 41 (2012) 5458-5460.

[15] J. Yao, L. Li, W.H. Benjamin Wong, C. Tan, D. Dong, H. Wang, Formation of ZIF-8 membranes and crystals in a diluted aqueous solution, Materials Chemistry and Physics, 139 (2013) 1003-1008.

[16] M.C. McCarthy, V. Varela-Guerrero, G.V. Barnett, H.K. Jeong, Synthesis of zeolitic imidazolate framework films and membranes with controlled microstructures, Langmuir, 26 (2010) 14636-14641.

[17] L. Kong, X. Zhang, H. Liu, J. Qiu, Synthesis of a highly stable ZIF-8 membrane on a macroporous ceramic tube by manual-rubbing $\mathrm{ZnO}$ deposition as a multifunctional layer, Journal of Membrane Science, 490 (2015) 354-363.

[18] Y.C. Pan, B. Wang, Z.P. Lai, Synthesis of ceramic hollow fiber supported zeolitic imidazolate framework-8 (ZIF-8) membranes with high hydrogen permeability, Journal of Membrane Science, 421 (2012) 292-298.

[19] M. Shah, H.T. Kwon, V. Tran, S. Sachdeva, H.K. Jeong, One step in situ synthesis of supported zeolitic imidazolate framework ZIF-8 membranes: Role of sodium formate, Microporous and Mesoporous Materials, 165 (2013) 63-69.

[20] C. Zhang, Y. Dai, J.R. Johnson, O. Karvan, W.J. Koros, High performance ZIF-8/6FDA-DAM mixed matrix membrane for propylene/propane separations, Journal of Membrane Science, 389 (2012) 34-42. 
[21] F. Cacho-Bailo, B. Seoane, C. Téllez, J. Coronas, ZIF-8 continuous membrane on porous polysulfone for hydrogen separation, Journal of Membrane Science, 464 (2014) 119-126.

[22] H. Bux, A. Feldhoff, J. Cravillon, M. Wiebcke, Y.-S. Li, J. Caro, Oriented Zeolitic Imidazolate Framework-8 Membrane with Sharp H2/C3H8 Molecular Sieve Separation, Chemistry of Materials, 23 (2011) 2262-2269.

[23] Y. Zhu, Q. Liu, J. Caro, A. Huang, Highly hydrogen-permselective zeolitic imidazolate framework ZIF-8 membranes prepared on coarse and macroporous tubes through repeated synthesis, Separation and Purification Technology, 146 (2015) 68-74.

[24] H.T. Kwon, H.K. Jeong, In situ synthesis of thin zeolitic-imidazolate framework ZIF-8 membranes exhibiting exceptionally high propylene/propane separation, J Am Chem Soc, 135 (2013) 10763-10768.

[25] H.T. Kwon, H.K. Jeong, Highly propylene-selective supported zeolite-imidazolate framework (ZIF-8) membranes synthesized by rapid microwave-assisted seeding and secondary growth, Chem Commun (Camb), 49 (2013) 3854-3856.

[26] D. Liu, X. Ma, H. Xi, Y.S. Lin, Gas transport properties and propylene/propane separation characteristics of ZIF-8 membranes, Journal of Membrane Science, 451 (2014) 85-93.

[27] Y.C. Pan, T. Li, G. Lestari, Z.P. Lai, Effective separation of propylene/propane binary mixtures by ZIF-8 membranes, Journal of Membrane Science, 390 (2012) 93-98.

[28] L. Diestel, H. Bux, D. Wachsmuth, J. Caro, Pervaporation studies of n-hexane, benzene, mesitylene and their mixtures on zeolitic imidazolate framework-8 membranes, Microporous and Mesoporous Materials, 164 (2012) 288-293.

[29] C. Zhang, R.P. Lively, K. Zhang, J.R. Johnson, O. Karvan, W.J. Koros, Unexpected Molecular Sieving Properties of Zeolitic Imidazolate Framework-8, The Journal of Physical Chemistry Letters, 3 (2012) 2130-2134.

[30] E.R. Nightingale, Phenomenological Theory of Ion Solvation. Effective Radii of Hydrated Ions, The Journal of Physical Chemistry, 63 (1959) 1381-1387.

[31] J. Jiang, Molecular simulations in metal-organic frameworks for divers potential applications, Molecular Simulation, 40 (2014) 21.

[32] Z. Hu, Y. Chen, J. Jiang, Zeolitic imidazolate framework-8 as a reverse osmosis membrane for water desalination: Insight from molecular simulation, The Journal of Chemical Physics, 134 (2011) 134705.

[33] M.C. Duke, B. Zhu, C.M. Doherty, M.R. Hill, A.J. Hill, M.A. Carreon, Structural effects on SAPO-34 and ZIF-8 materials exposed to seawater solutions, and their potential as desalination membranes, Desalination, 377 (2016) 128-137.

[34] Y. Zhu, K.M. Gupta, Q. Liu, J. Jiang, J. Caro, A. Huang, Synthesis and seawater desalination of molecular sieving zeolitic imidazolate framework membranes, Desalination, 385 (2016) 75-82.

[35] Y.S. Lin, Metal organic framework membranes for separation applications, Current Opinion in Chemical Engineering, 8 (2015) 21-28.

[36] X.L. Liu, Y.S. Li, Y.J. Ban, Y. Peng, H. Jin, H. Bux, L.Y. Xu, J. Caro, W.S. Yang, Improvement of hydrothermal stability of zeolitic imidazolate frameworks, Chemical Communications, 49 (2013) 9140-9142.

[37] Schweinefu, S. Springer, I.A. Baburin, T. Hikov, K. Huber, S. Leoni, M. Wiebcke, Zeolitic imidazolate framework-71 nanocrystals and a novel SOD-type polymorph: solution mediated phase transformations, phase selection via coordination modulation and a density functional theory derived energy landscape, Dalton Transactions, 43 (2014) 3528-3536.

[38] A. Kasik, X. Dong, Y.S. Lin, Synthesis and stability of zeolitic imidazolate framework-68 membranes, 
Microporous and Mesoporous Materials, 204 (2015) 99-105.

[39] H. Zhang, D. Liu, Y. Yao, B. Zhang, Y.S. Lin, Stability of ZIF-8 membranes and crystalline powders in water at room temperature, Journal of Membrane Science, 485 (2015) 103-111.

[40] H. Yin, H. Kim, J. Choi, A.C.K. Yip, Thermal stability of ZIF-8 under oxidative and inert environments: A practical perspective on using ZIF-8 as a catalyst support, Chemical Engineering Journal, 278 (2015) 293-300.

[41] J.B. James, Y.S. Lin, Kinetics of ZIF-8 Thermal Decomposition in Inert, Oxidizing, and Reducing Environments, The Journal of Physical Chemistry C, (2016).

[42] J.A. Thompson, C.R. Blad, N.A. Brunelli, M.E. Lydon, R.P. Lively, C.W. Jones, S. Nair, Hybrid Zeolitic Imidazolate Frameworks: Controlling Framework Porosity and Functionality by Mixed-Linker Synthesis, Chemistry of Materials, 24 (2012) 1930-1936.

[43] O. Karagiaridi, M.B. Lalonde, W. Bury, A.A. Sarjeant, O.K. Farha, J.T. Hupp, Opening ZIF-8: A Catalytically Active Zeolitic Imidazolate Framework of Sodalite Topology with Unsubstituted Linkers, Journal of the American Chemical Society, 134 (2012) 18790-18796.

[44] H. Fei, J.F. Cahill, K.A. Prather, S.M. Cohen, Tandem Postsynthetic Metal Ion and Ligand Exchange in Zeolitic Imidazolate Frameworks, Inorganic Chemistry, 52 (2013) 4011-4016.

[45] S. Park, W.R. Kang, H.T. Kwon, S. Kim, M. Seo, J. Bang, S.h. Lee, H.K. Jeong, J.S. Lee, The polymeric upper bound for N2/NF3 separation and beyond; ZIF-8 containing mixed matrix membranes, Journal of Membrane Science, 486 (2015) 29-39. 